\title{
Magnetic resonance imaging before breast cancer surgery: results of an observational multicenter international prospective analysis (MIPA)
}

\author{
Francesco Sardanelli ${ }^{1,2}$ - Rubina M. Trimboli ${ }^{1}$ Nehmat Houssami ${ }^{3}$. Fiona J. Gilbert ${ }^{4} \cdot$ Thomas H. Helbich $^{5}$. \\ Marina Álvarez Benito ${ }^{6}$. Corinne Balleyguier ${ }^{7} \cdot$ Massimo Bazzocchi $^{8} \cdot$ Peter Bult $^{9} \cdot$ Massimo Calabrese $^{10}$. \\ Julia Camps Herrero ${ }^{11}$. Francesco Cartia ${ }^{12}$. Enrico Cassano ${ }^{13}$. Paola Clauser ${ }^{5}$. Andrea Cozzi ${ }^{1}$. \\ Danúbia A. de Andrade ${ }^{14}$. Marcos F. de Lima Docema ${ }^{15}$. Catherine Depretto ${ }^{12}$. Valeria Dominelli ${ }^{13}$. Gábor Forrai ${ }^{16}$. \\ Rossano Girometti ${ }^{8}$. Steven E. Harms ${ }^{17}$. Sarah Hilborne ${ }^{4}$ - Raffaele lenzi ${ }^{18}$. Marc B. I. Lobbes ${ }^{19}$. Claudio Losio ${ }^{20}$. \\ Ritse M. Mann ${ }^{21,22}$. Stefania Montemezzi ${ }^{23}$. Inge-Marie Obdeijn ${ }^{24}$. Umit A. Ozcan ${ }^{25}$. Federica Pediconi ${ }^{26}$. \\ Katja Pinker ${ }^{5,27}$. Heike Preibsch ${ }^{28}$. José L. Raya Povedano ${ }^{6}$. Daniela Sacchetto ${ }^{29,30,31}$. Gianfranco P. Scaperrotta ${ }^{12}$. \\ Simone Schiaffino ${ }^{2} \cdot$ Margrethe Schlooz ${ }^{32}$ - Botond K. Szabó ' $^{33}$. Donna B. Taylor ${ }^{34,35}$. Özden S. Ulus ${ }^{25}$. \\ Mireille Van Goethem ${ }^{36}$. Jeroen Veltman ${ }^{37} \cdot$ Stefanie Weigel $^{38} \cdot$ Evelyn Wenkel $^{39} \cdot$ Chiara Zuiani $^{8} \cdot$ Giovanni Di Leo $^{2}$
}

Received: 5 June 2021 / Revised: 20 July 2021 / Accepted: 2 August 2021 / Published online: 13 October 2021

(C) The Author(s) 2021, corrected publication 2022

\begin{abstract}
Objectives Preoperative breast magnetic resonance imaging (MRI) can inform surgical planning but might cause overtreatment by increasing the mastectomy rate. The Multicenter International Prospective Analysis (MIPA) study investigated this controversial issue. Methods This observational study enrolled women aged 18-80 years with biopsy-proven breast cancer, who underwent MRI in addition to conventional imaging (mammography and/or breast ultrasonography) or conventional imaging alone before surgery as routine practice at 27 centers. Exclusion criteria included planned neoadjuvant therapy, pregnancy, personal history of any cancer, and distant metastases.

Results Of 5896 analyzed patients, 2763 (46.9\%) had conventional imaging only (noMRI group), and 3133 (53.1\%) underwent MRI that was performed for diagnosis, screening, or unknown purposes in 692/3133 women (22.1\%), with preoperative intent in 2441/3133 women (77.9\%, MRI group). Patients in the MRI group were younger, had denser breasts, more cancers $\geq 20 \mathrm{~mm}$, and a higher rate of invasive lobular histology than patients who underwent conventional imaging alone ( $p<0.001$ for all comparisons). Mastectomy was planned based on conventional imaging in 22.4\% (MRI group) versus $14.4 \%$ (noMRI group) $(p<0.001)$. The additional planned mastectomy rate in the MRI group was $11.3 \%$. The overall performed first- plus second-line mastectomy rate was 36.3\% (MRI group) versus $18.0 \%$ (noMRI group) $(p<0.001$ ). In women receiving conserving surgery, MRI group had a significantly lower reoperation rate $(8.5 \%$ versus $11.7 \%, p<0.001)$.

Conclusions Clinicians requested breast MRI for women with a higher a priori probability of receiving mastectomy. MRI was associated with $11.3 \%$ more mastectomies, and with 3.2\% fewer reoperations in the breast conservation subgroup.

Key Points

- In 19\% of patients of the MIPA study, breast MRI was performed for screening or diagnostic purposes.

- The current patient selection to preoperative breast MRI implies an $11 \%$ increase in mastectomies, counterbalanced by a $3 \%$ reduction of the reoperation rate.

- Data from the MIPA study can support discussion in tumor boards when preoperative MRI is under consideration and should be shared with patients to achieve informed decision-making.
\end{abstract}

Keywords Breast cancer $\cdot$ Magnetic resonance imaging $\cdot$ Mastectomy $\cdot$ Breast-conserving surgery $\cdot$ Reoperation

Francesco Sardanelli

francesco.sardanelli@unimi.it

Extended author information available on the last page of the article 


\section{Abbreviations}

ACR American College of Radiology

CI Confidence interval

MRI Magnetic resonance imaging

OR Odds ratio

\section{Introduction}

In patients newly diagnosed with breast cancer, the routine use of breast magnetic resonance imaging (MRI) before surgery is a controversial topic [1,2], attracting extensive debate and little consensus [3-5]. Proponents reasonably draw on the established evidence of MRI sensitivity to detect additional disease, allowing more tailored surgical planning $[6,7]$. Opponents point out the lack of evidence on clinical benefit from preoperative MRI and raise concerns that it causes more mastectomies than needed [8-10]. Guidelines are heterogenous, ranging from defined but limited indications $[11,12]$ to recommendations against [13].

The Multicenter International Prospective Analysis (MIPA) study was undertaken to provide new knowledge on this topic, building evidence on whether and to what extent MRI impacts surgical treatment in breast cancer practice.

\section{Methods}

\section{Study design and population}

The MIPA study was registered in the International Standard Randomized Controlled Trial Number Register (ISRCTN41143178). Methods, detailed in the protocol paper [14], are summarized here.

The study was initiated by the European Network for the Assessment of Imaging in Medicine, endorsed by the European Society of Breast Imaging, and conducted in accordance with the Declaration of Helsinki. It was approved on January 29, 2013, by the Ethics Committee of the coordinating center (protocol number 2784) and thereafter by local Ethics Committees of participating centers. All participants signed an informed consent, unless waived by local Ethics Committees.

The study was coordinated and monitored by the principal investigator at IRCCS Policlinico San Donato, San Donato Milanese, Italy. The principal investigator (first author) and the lead statistician (last author) had full access to the database, generated statistical analyses, prepared the first manuscript draft, and assume responsibility for the accuracy and completeness of the data and for the adherence of the study to the protocol.
MIPA is a large-scale observational study enrolling women with needle biopsy-proven breast cancer. Enrolled patients underwent or did not undergo MRI before surgery as part of routine practice at each center, resulting in two concurrent groups ex post: women who underwent digital mammography/tomosynthesis and/or breast ultrasonography, i.e., conventional imaging (noMRI group), and women who received MRI in addition to conventional imaging (MRI group). Following a public call, centers were selected for participation among those that documented high breast MRI volumes and the use of protocols recommended by international societies $[11,12,15]$. All MRI examinations were performed before/after administration of macrocyclic or linear contrast agents (in Europe, only before the linear ones were banned). Gadobutrol (Bayer AG) at a dose of $0.1-0.2 \mathrm{mmol} / \mathrm{kg}$ of body weight was used in $22 / 27$ centers.

The study population included women aged 18-80 years newly diagnosed with breast cancer and amenable to upfront surgery. Exclusion criteria were an indication to neoadjuvant therapy, personal history of breast or any other cancer, distant metastases at diagnosis, pregnancy, and inability to provide consent.

MRI examinations were classified as performed with preoperative (ipsilateral local staging and contralateral screening), diagnostic (problem-solving), or bilateral screening purposes.

\section{Endpoints}

Primary endpoints were the first-line mastectomy rate (endpoint 1) and the immediate/short term reoperation rate for close or positive margins (endpoint 2), calculated amongst all patients. Secondary surgical endpoints were the first-line only bilateral mastectomy rate (endpoint 3 ), and the overall mastectomy rate (endpoint 4), obtained summing first-line mastectomies and conserving surgeries converted into mastectomies. For the MRI group, other secondary surgical endpoints were the additional mastectomy rate and the rate of change to more or less extensive conserving surgery (wider excision or multiple excisions). Secondary clinical endpoints (rate of breast recurrence and distant metastases) will be evaluated at a 5-year follow-up.

\section{Sample size and statistical analysis}

The sample size calculation, set at 7000 patients, has been described in the protocol paper [14].

Statistical analysis followed a per-protocol approach. Only patients having an electronic case report form with all data needed for the endpoints' calculation were analyzed. Statistical analysis was performed on a per-patient basis 
whenever possible. In this regard, patients receiving mastectomy on one side and conserving surgery on the other side, as well as those receiving bilateral mastectomy, were considered as mastectomy patients.

The MRI and noMRI groups were compared in terms of baseline characteristics including demographics, high familial breast cancer risk (three or more first-degree relatives with breast or ovarian cancer), breast density, tumor size, histopathology at needle biopsy, and surgical plan based on conventional imaging only, as defined by the multidisciplinary team. To allow a reasonable comparison between the two groups, patients receiving MRI for screening or diagnostic purposes were excluded from comparative analysis, since surgical planning based on conventional imaging was not possible.

Given that MIPA is a nonrandomized study, the inferential analysis used statistical tests to ascertain whether the two groups were homogenous. Depending on distributions, comparisons of continuous variables were performed using the Student $t$ test or the Mann-Whitney $U$ test for independent data, or using the $\chi^{2}$ test for categorical variables.

Baseline characteristics were included as covariates in statistical modelling. A binary logistic regression analysis was performed using Nagelkerke $R^{2}$ as a measure of the endpoint variability that was explained by the analyzed predictors. Predictors were added one by one and kept in the final model only if increasing $R^{2}$ or if the associated $p$ value was $<0.05$. Odds ratios (ORs) for such predictors with their 95\% CIs were calculated. The final model's area under the curve was calculated using receiver operating characteristic analysis.

In the MRI group, the mastectomy rate planned before and after MRI was calculated: the difference was the measure of the additional mastectomy rate associated with MRI.

\section{Results}

Patient enrollment began in June 2013 and ended in November 2018, achieving a total of 7245 patients in 27 participating centers worldwide, enrollment per center being detailed in the protocol paper [14]. Of the 7245 enrolled patients, $1349(18.6 \%)$ were excluded a priori from analysis, being screening failures (i.e., one or more exclusion criteria discovered only after enrollment), treated with neoadjuvant therapy after enrollment, patients who moved to another center, or patients who had missing data. Among the remaining 5896 patients, 3133/5896 (53.1\%) underwent MRI in addition to conventional imaging, with the following purposes: diagnostic in $496 / 3133$ women $(15.8 \%)$, bilateral screening in $111 / 3133$ women (3.5\%), unknown in $85 / 3133$ women (2.8\%), and explicitly preoperative in $2441 / 3133$ women $(77.9 \%)$, this last group being the MRI group considered for comparison. On the other hand, 2763/5896 women $(46.9 \%)$ received conventional imaging only (noMRI group). Preoperative MRI was ordered by: radiologists alone in 1309/2441 women (53.6\%); surgeons alone in 808/2441 women $(33.1 \%)$; radiologists and surgeons in $216 / 2441$ women $(8.8 \%)$; radiologists, surgeons, and oncologists in $65 / 2441$ women $(2.7 \%)$; other combinations of physicians in $43 / 2441$ women $(1.8 \%)$. The enrolment flowchart is shown in Fig. 1.

\section{Between-group comparison of baseline characteristics}

Table 1 reports differences in characteristics between groups. The MRI group included younger women $(57 \pm 11$ versus $61 \pm 11$ years, $p<0.001)$ and a larger proportion of pre- or perimenopausal women $(40.0 \%$ versus $26.6 \%$, $p<0.001)$, women with dense breasts $(50.1 \%$ versus $33.2 \%$, $p<0.001$ ), invasive lobular histology at percutaneous biopsy ( $17.4 \%$ versus $7.9 \%, p<0.001)$, and cancers $\geq 20 \mathrm{~mm}$ on final pathology $(42.4 \%$ versus $27.5 \%, p<0.001)$.

\section{Planned mastectomy}

Mastectomy planned on the basis of conventional imaging only was more frequent in the MRI group (22.4\% versus $14.4 \%$ ), with a crude OR of 1.7 (95\% CI, 1.5 to 2.0 ). Similarly, bilateral mastectomy was planned on the basis of conventional imaging in $31 / 516$ women $(6.0 \%)$ of the MRI group and in $8 / 398$ women $(2.0 \%)$ of the noMRI group, with a crude OR of 2.9 (95\% CI, 1.3 to 6.4 ).

In the MRI group, mastectomy was already planned on conventional imaging in 547/2441 women $(22.4 \%)$, in 791 women after MRI (32.4\%), and was ultimately performed in 823/2441 women (33.7\%) (Fig. 2). These 276 additional mastectomies (from 547 to 823 ) in the MRI group included patient's preference in 50/2441 women (2.0\%) and recommendations from surgeons and/or other physicians in $7 / 2441$ women $(0.3 \%)$ (Fig. 3). Moreover, this $11.3 \%$ additional mastectomy absolute rate associated with MRI (from 22.4 to $33.7 \%$ ) was the difference between an $11.6 \%$ rate of conversion from conserving surgery to mastectomy (284/2441 women) and a $0.3 \%$ rate of opposite conversion, from mastectomy to conserving surgery ( $8 / 2441$ women). The $284 / 2441$ (11.6\%) conversions from conserving surgery to mastectomy were prompted in $223 / 2441$ cases by additional MRI findings $(9.1 \%)$ and by other reasons in $61 / 2441$ cases $(2.5 \%)$, mainly dominated by patient's preference (50/2441 cases, $2.0 \%$ ). As shown in Fig. 3, these conversions were supported by malignancy at needle sampling in $80 / 223$ cases $(35.9 \%)$, with $67 / 131(51.1 \%)$ cases in which needle sampling was not 


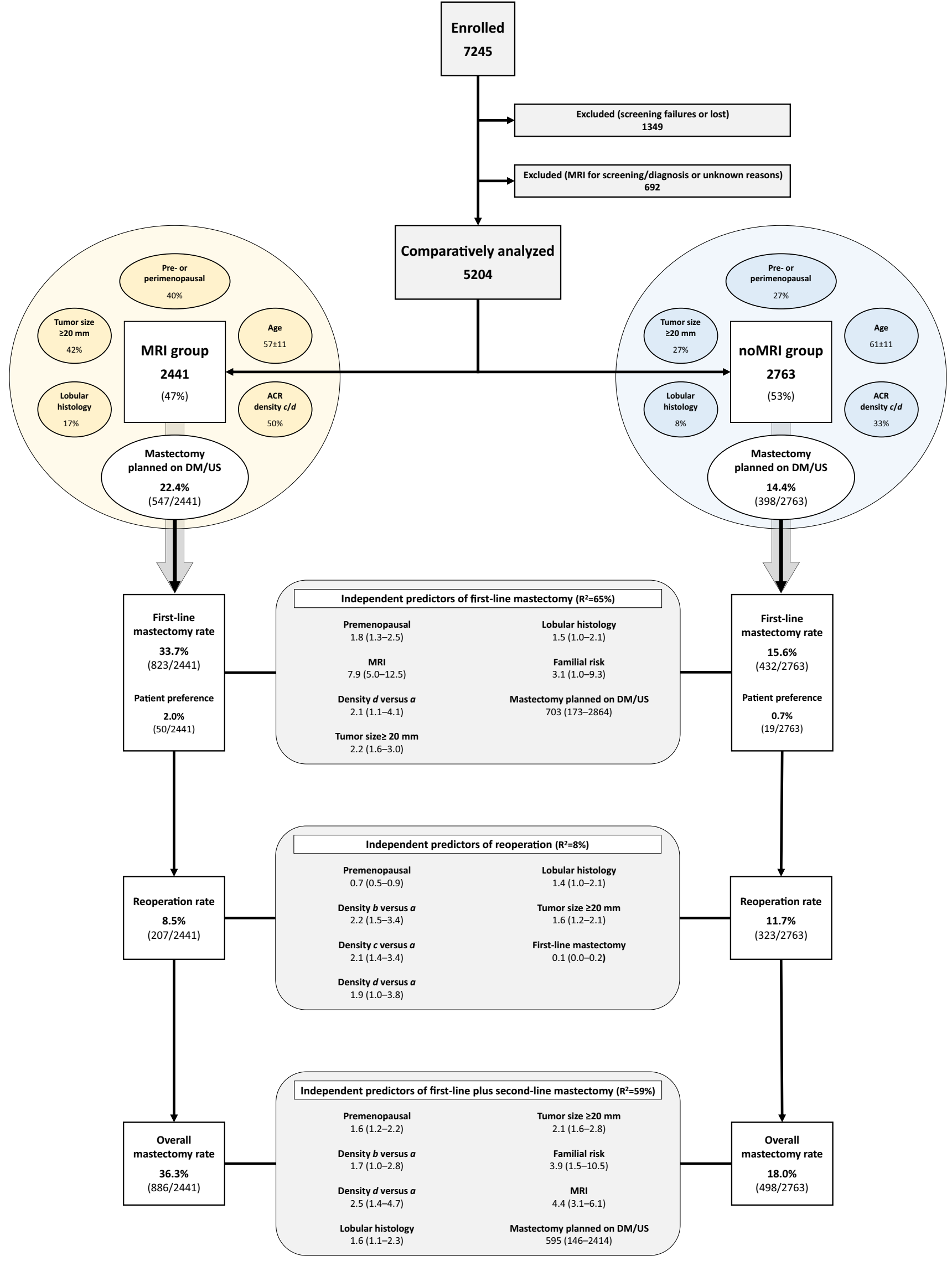


४Fig. 1 Study flowchart and results regarding predictors of surgical outcome. For each predictor, odds ratio and 95\% confidence interval are reported. $R^{2}$ represents the Nagelkerke goodness of fit. MRI, magnetic resonance imaging; DM, digital mammography; US, ultrasonography

performed being found to be multifocal or multicentric on final pathology.

Considering the 1641 breasts that received conserving surgery in the MRI group (with available data for this analysis), the surgical extent was as planned on conventional imaging in $1387 / 1641$ cases $(84.5 \%)$. The remaining 254 women received less extensive surgery $(26 / 1641$, $1.6 \%)$, more extensive single excision $(186 / 1641,11.3 \%)$, or multiple excisions (42/1641, 2.6\%).

\section{First-line unilateral and bilateral mastectomy}

In the noMRI group, the rate of mastectomies that were actually performed as first-line surgery (endpoint 1) increased from the $14.4 \%$ planned on conventional imaging to the $15.6 \%$ that was actually performed. The MRI group had a more than double mastectomy rate compared to the noMRI group (33.7\% versus $15.6 \%$ ), with a crude OR of 2.7 (95\% CI, 2.4 to 3.1). These percentages include the patient's preference to receive mastectomy: $2.0 \%$ in the MRI group and $0.7 \%$ in the noMRI group. Logistic regression analysis (Fig. 1 and Table 2) showed that MRI was an independent risk factor for mastectomy (OR 7.9), together with the highest breast density (American College of Radiology [ACR] category $d$ versus category $a$, OR 2.1), invasive lobular histology at biopsy (OR 1.5), high familial risk (OR 3.1), premenopausal status (OR 1.8 ), lesion diameter $\geq 20 \mathrm{~mm}$ (OR 2.2), and planned mastectomy on conventional imaging (OR 703). The area under the curve of this model was 0.914 .

The rate of first-line bilateral mastectomy among women receiving mastectomy (endpoint 3) was $87 / 823$ (10.6\%) in the MRI group (30/823 cases, 3.6\%, due to patient's preference) and $12 / 432(2.8 \%)$ in the noMRI group (2/432 cases, $0.5 \%$, due to patient's preference), with a crude OR of 4.1 (95\% CI, 2.2 to 7.7$)$. In the logistic regression analysis (Table 3), MRI (OR 3.6) and high familial risk (OR 4.7) were the only independent risk factors for bilateral mastectomy, while mastectomy planned on conventional imaging was a protective factor (OR 0.6).

\section{Reoperation and overall mastectomy rate}

The reoperation rate for close/positive margins (endpoint 2) was lower in the MRI group than in the noMRI group $(8.5 \%$ versus $11.7 \%, p<0.001)$, with a crude OR of $0.70(95 \% \mathrm{CI}$,
0.58 to 0.84 ). In the logistic regression analysis (Fig. 1 and Table 4), breast density was a risk factor for reoperation: compared to ACR density $a$, OR was 2.2 for $b, 2.1$ for $c$, and 1.9 for $d$. Invasive lobular histology (OR 1.4) as well as lesion diameter $\geq 20 \mathrm{~mm}$ were also independent risk factors (OR 1.6). Vice versa, premenopausal status (OR 0.7) and received mastectomy as first-line surgery (OR 0.1 ) were protective factors against reoperation.

The overall mastectomy rate (endpoint 4) was higher in the MRI group than in the noMRI group (36.3\% versus $18.0 \%$ ), with a crude OR of 2.6 (95\% CI, 2.3 to 2.9 ). As shown in Fig. 1 and Table 5, premenopausal status (OR 1.6), breast density (OR 1.7 for ACR density $b$ and 2.5 for $d$, compared to ACR density $a$ ) high familial risk (OR 3.9), invasive lobular histology (OR 1.6), MRI (OR 4.4), lesion diameter $\geq 20 \mathrm{~mm}$ (OR 2.1), and planned mastectomy on conventional imaging (OR 595) increased the odds of mastectomy.

\section{Discussion}

This study reports actual clinical practice with breast MRI before breast cancer surgery. Of 5896 patients, about half received MRI. However, MRI was performed for screening, diagnosis, or unknown purpose in 692/5896 patients (over one in five patients), i.e., not originally intended as "preoperative." In the $2441 / 5896$ patients having MRI performed with preoperative intent (MRI group, 41.4\%), MRI was requested by radiologists alone $(53.6 \%)$ or surgeons alone $(33.1 \%)$, reflecting current clinical practice [16, 17]. MRI was preferentially ordered for younger and premenopausal patients, for patients with dense breast tissue, with lobular cancers, or cancers $\geq 20 \mathrm{~mm}$, as a survey already suggested [17].

Our data outline the contribution of conventional imaging to mastectomy indication in patients who also had MRI and define factors predicting surgical outcomes, including but not limited to MRI. Considering the overall (first- plus second-line) mastectomy rate, the higher rate $(36.3 \%)$ found in the MRI group compared to the noMRI group (18.0\%) represents a major finding for surgical oncology. The role of premenopausal status, breast density, familial risk, cancer diameter $\geq 20 \mathrm{~mm}$, and invasive lobular histology in increasing the mastectomy risk (see Table 1) was expected [18, 19]. Importantly, a planned mastectomy on conventional imaging inherently made mastectomy almost unavoidable, with all but eight conventional imaging-based mastectomies confirmed after MRI. Conventional imaging had already suggested mastectomy in $66.5 \%$ of the women who ultimately received such surgery, showing that MRI was often used as a confirmation tool. Indeed, women with a mastectomy planned on conventional imaging had a 1.7-fold probability to receive MRI compared to those with a conserving surgery 
Table 1 Patient and tumor characteristics in the MIPA study

\begin{tabular}{|c|c|c|c|}
\hline Patient or tumor characteristic & MRI group & noMRI group & $p$ \\
\hline Age at diagnosis-years ${ }^{\mathrm{a}}$ & $57 \pm 11$ & $61 \pm 11$ & $<0.001$ \\
\hline Pre- or perimenopausal (\%) & $\begin{array}{l}972 / 2432 \\
(40.0 \%)\end{array}$ & $\begin{array}{l}731 / 2748 \\
(26.6 \%)\end{array}$ & $<0.001$ \\
\hline Dense breast: ACR density category $c$ or $d(\%)$ & $\begin{array}{l}1171 / 2336 \\
(50.1 \%)\end{array}$ & $\begin{array}{l}831 / 2503 \\
(33.2 \%)\end{array}$ & $<0.001$ \\
\hline $\begin{array}{l}\text { Three or more first-degree relatives with history of breast } \\
\text { or ovarian cancer }(\%)\end{array}$ & $\begin{array}{l}35 / 2432 \\
(1.4 \%)\end{array}$ & $\begin{array}{l}34 / 2749 \\
(1.2 \%)\end{array}$ & 0.526 \\
\hline BRCA1/2 mutation & $\begin{array}{l}20 / 2431 \\
(0.8 \%)\end{array}$ & $\begin{array}{l}14 / 2748 \\
(0.5 \%)\end{array}$ & 0.161 \\
\hline Multifocal/multicentric breast cancer on $\mathrm{DM}^{\mathrm{b}}(\%)$ & $\begin{array}{l}307 / 2143 \\
(14.3 \%)\end{array}$ & $\begin{array}{l}212 / 2435 \\
(8.7 \%)\end{array}$ & $<0.001$ \\
\hline Diameter of the largest lesion on DM and/or US- $\mathrm{mm}^{\mathrm{c}}$ & $\begin{array}{l}16 \\
\text { (11 to } 24)\end{array}$ & $\begin{array}{l}15 \\
\text { (10 to } 21)\end{array}$ & $<0.001$ \\
\hline Ductal carcinoma in situ at biopsy (\%) & $\begin{array}{l}409 / 2421 \\
(16.9 \%)\end{array}$ & $\begin{array}{l}472 / 2554 \\
(18.5 \%)\end{array}$ & 0.255 \\
\hline Invasive lobular histology at biopsy (\%) & $\begin{array}{l}341 / 1959 \\
(17.4 \%)\end{array}$ & $\begin{array}{l}164 / 2078 \\
(7.9 \%)\end{array}$ & $<0.001$ \\
\hline Triple-negative breast cancer (\%) & $\begin{array}{l}103 / 2234 \\
(4.6 \%)\end{array}$ & $\begin{array}{l}107 / 2391 \\
(4.5 \%)\end{array}$ & 0.585 \\
\hline Tumor size on final pathology $\geq 20 \mathrm{~mm}(\%)$ & $\begin{array}{l}799 / 1886 \\
(42.4 \%)\end{array}$ & $\begin{array}{l}537 / 1953 \\
(27.5 \%)\end{array}$ & $<0.001$ \\
\hline
\end{tabular}

Distributions are given per patient, unless differently specified. Variations of denominators are due to different rates of unknown data

ACR American College of Radiology; DM digital mammography; US ultrasound

${ }^{a}$ Mean \pm standard deviation

${ }^{\mathrm{b}}$ Per-breast distribution

${ }^{\mathrm{c}}$ Per-breast distribution, given as median and interquartile interval planned on conventional imaging, while the additional firstline mastectomy rate after MRI was curtailed to $11.3 \%$.

In addition, the higher first-line bilateral mastectomy rate in the MRI group (10.6\% versus 2.8\%), decreasing to $7.0 \%$ versus $2.3 \%$ when subtracting bilateral surgeries due to patients' preference, was partially driven by patient selection. Indeed, logistic regression analysis confirmed the role of both familial risk and MRI in predicting first-line bilateral mastectomy, with an OR of 4.7 and 3.6, respectively. Moreover, mastectomy planned on conventional imaging acted as a protective factor against first-line bilateral mastectomy. These findings derive from the high MRI sensitivity also for contralateral lesions and from a selection to MRI of patients with a higher propensity to bilateral mastectomy. Indeed, women with a planned bilateral mastectomy on conventional imaging had a 2.9 -fold probability of receiving MRI compared to those with unilateral mastectomy planned on conventional imaging.

Regarding conversions from conserving surgery to mastectomy attributed to additional MRI findings, we note that only 80/223 (35.9\%) cases were confirmed as malignant by needle sampling, with other $5 / 223(2.2 \%)$ cases being reported as negatives. For the remaining 138/223 cases (61.9\%), we must consider the crucial point of an accurate three-dimensional radiological-pathological correlation between MRI findings and overall mastectomy specimens, a task very difficult to be performed in real-world clinical practice [20].

In our study, the MRI group had an absolute 3.2\% lower reoperation rate compared to the noMRI group $(8.5 \%$ and $11.7 \%$, respectively), as already hinted by two randomized controlled trials [21, 22]. The POMB trial [21], investigating a relatively young population, reported a significant reduction in reoperation rate from 15 to $5 \%$, while the IRCIS trial [22], assessing ductal carcinoma in situ, reported a non-significant reduction from 27 to $20 \%$. Breast density, invasive lobular histology, and diameter $\geq 20 \mathrm{~mm}$ negatively impacted our overall reoperation rate, as already reported by other authors [23-25]. Vice versa, premenopausal status and, again as expected, first-line mastectomy reduced the odds for reoperation. However, MRI did not act as an independent reducer of reoperation risk.

Hence, the MIPA study brings new insights in comparison with previous randomized studies $[26,27]$ and meta-analyses [4] which did not report any reduction in reoperation rate in their MRI groups. However, the reduction in the reoperation rate demonstrated in our MRI group must be read in the light of the increase in mastectomy 


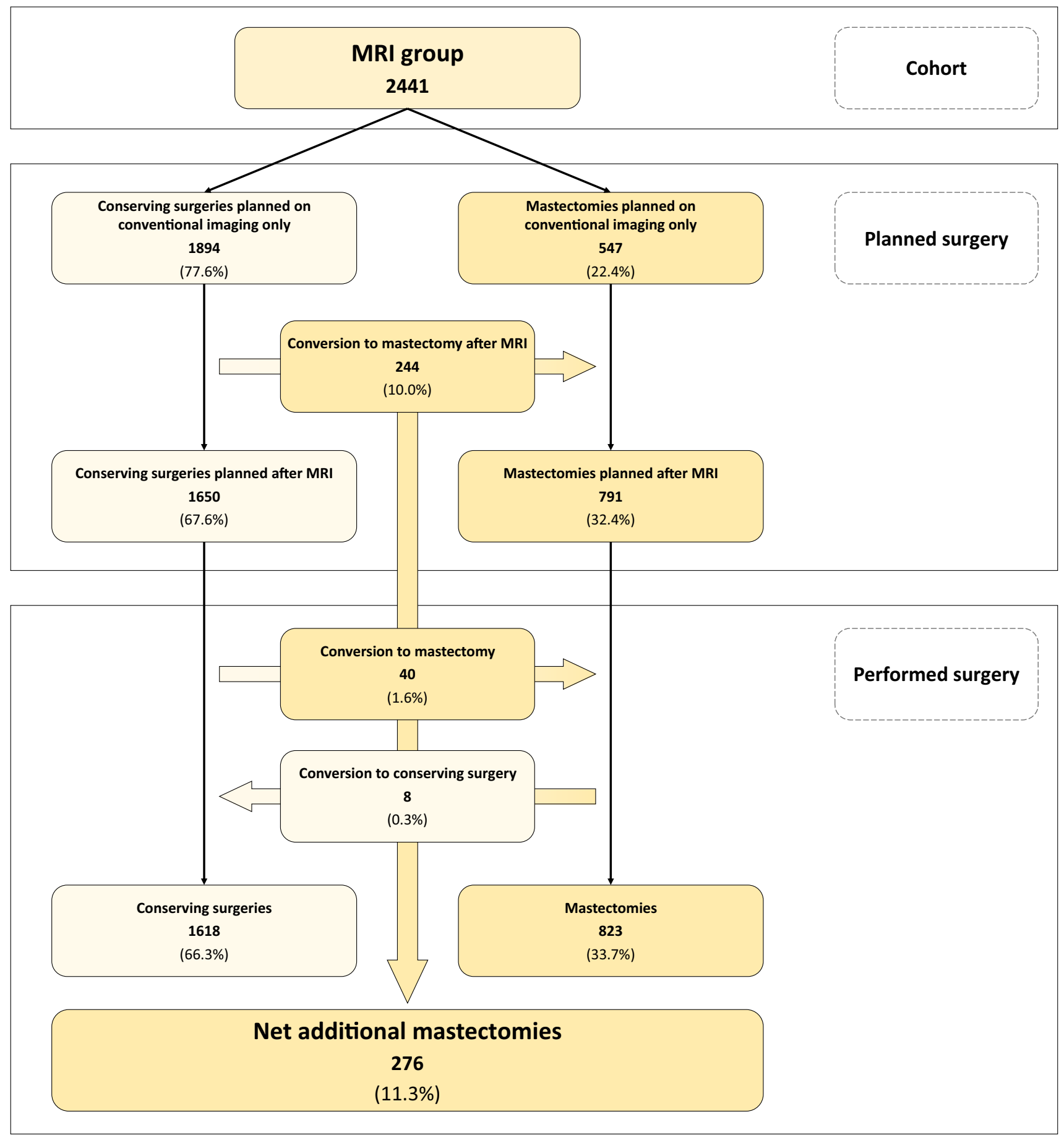

Fig. 2 Surgical planning and performed surgery in the MRI group. MRI, magnetic resonance imaging

rate, as shown by the protective role of the first-line mastectomy against reoperation. This reasoning also applies to our finding that mastectomy planned on conventional imaging protected against reoperation. The trade-off between first-line mastectomy and reoperation is a matter for debate with relevant issues in terms of psychological impact, complication rates, and final cosmetic results [28,
29]. Regarding conserving surgery, MRI did not alter the surgical planning based on conventional imaging in $84.5 \%$ of cases. MRI-based conserving surgery was more extensive than planned on conventional imaging in $13.9 \%$ and less extensive in $1.6 \%$ of cases. The question of whether MRI-tailored surgery will benefit patients at follow-up awaits future evaluation of our MIPA cohorts, including 


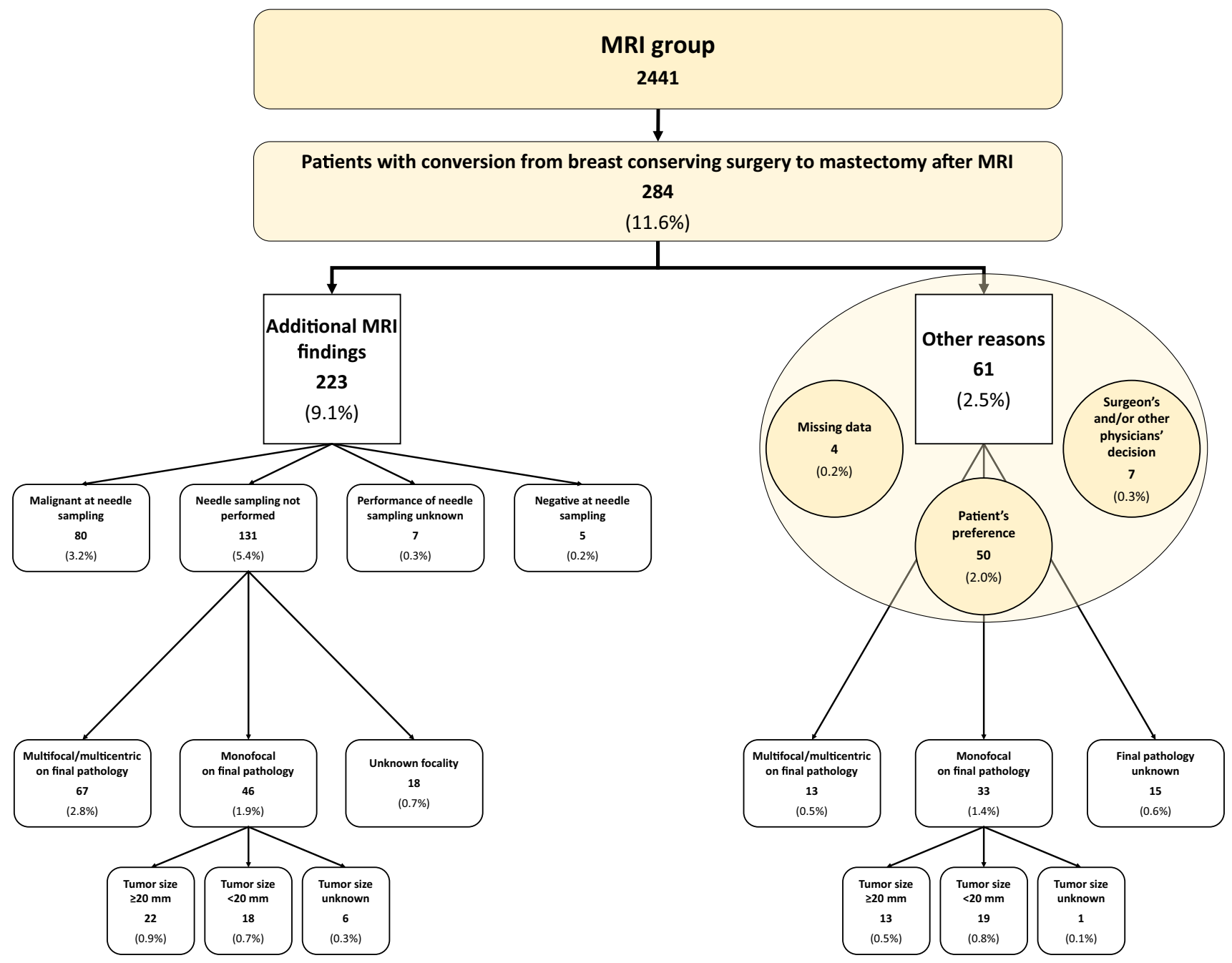

Fig. 3 Reasons for conversion from breast-conserving surgery to mastectomy in the MRI group. MRI, magnetic resonance imaging

Table 2 Logistic regression model of variables associated with first-line mastectomy

\begin{tabular}{lllrcc}
\hline Variable & T & Standard error & Wald & $p$ & Odds ratio (95\% CI) \\
\hline MRI & 2.071 & 0.230 & 80.780 & $<0.001$ & $7.93(5.05-12.46)$ \\
High familial risk $^{\mathrm{a}}$ & 1.133 & 0.560 & 4.097 & 0.043 & $3.10(1.04-9.29)$ \\
Premenopausal & 0.560 & 0.172 & 10.550 & 0.001 & $1.75(1.25-2.46)$ \\
ACR breast density category $b$ & 0.199 & 0.275 & 0.524 & 0.469 & $1.22(0.71-2.09)$ \\
ACR breast density category $c$ & 0.151 & 0.287 & 0.277 & 0.599 & $1.16(0.66-2.04)$ \\
ACR breast density category $d$ & 0.762 & 0.331 & 5.302 & 0.021 & $2.14(1.12-4.10)$ \\
Lobular histology & 0.372 & 0.198 & 3.519 & 0.061 & $1.45(0.98-2.14)$ \\
Tumor size on final pathology $\geq 20$ mm & 0.781 & 0.156 & 25.174 & $<0.001$ & $2.18(1.61-2.96)$ \\
Planned mastectomy on conventional imaging & 6.556 & 0.716 & 83.784 & $<0.001$ & $703.47(172.81-2863.61)$ \\
Constant & -4.685 & 0.308 & 231.567 & $<0.001$ & 0.009 \\
\hline
\end{tabular}

Nagelkerke $R^{2}=65.3 \%$. Area under the curve $=0.914$

ACR American College of Radiology; CI confidence interval; MRI magnetic resonance imaging

a Three or more first-degree relatives with breast or ovarian cancer 
Table 3 Variables associated with first-line bilateral mastectomy in the subgroup of breast cancer patients receiving first-line mastectomy

\begin{tabular}{|c|c|c|c|c|c|}
\hline Variable & $\mathrm{T}$ & Standard error & Wald & $p$ & Odds ratio $(95 \% \mathrm{CI})$ \\
\hline MRI & 1.291 & 0.323 & 15.960 & $<0.001$ & $3.64(1.93-6.85)$ \\
\hline High familial risk ${ }^{\mathrm{a}}$ & 1.554 & 0.471 & 10.905 & 0.001 & $4.73(1.88-11.90)$ \\
\hline $\begin{array}{c}\text { Planned mastectomy on } \\
\text { conventional imaging }\end{array}$ & -0.508 & 0.223 & 5.193 & 0.023 & $0.60(0.38-0.93)$ \\
\hline Constant & -3.165 & 0.354 & 80.058 & $<0.001$ & 0.042 \\
\hline
\end{tabular}

Nagelkerke $R^{2}=7.9 \%$. Area under the curve $=0.669$

CI confidence interval; $M R I$ magnetic resonance imaging

${ }^{a}$ Three or more first-degree relatives with breast or ovarian cancer

\begin{tabular}{lllrrr}
\hline Variable & T & $\begin{array}{l}\text { Standard } \\
\text { error }\end{array}$ & \multicolumn{1}{l}{ Wald } & \multicolumn{1}{l}{ Odds ratio (95\% CI) } \\
\hline Premenopausal & -0.428 & 0.165 & 6.709 & 0.010 & $0.65(0.47-0.90)$ \\
ACR breast density category $b$ & 0.788 & 0.216 & 13.244 & $<0.001$ & $2.20(1.44-3.36)$ \\
ACR breast density category $c$ & 0.758 & 0.232 & 10.646 & 0.001 & $2.14(1.35-3.37)$ \\
ACR breast density category $d$ & 0.636 & 0.351 & 3.289 & 0.070 & $1.89(0.95-3.76)$ \\
Lobular histology & 0.360 & 0.183 & 3.868 & 0.049 & $1.43(1.00-2.05)$ \\
Tumor size on final pathology $\geq 20 \mathrm{~mm}$ & 0.460 & 0.137 & 11.314 & 0.001 & $1.58(1.21-2.07)$ \\
First-line mastectomy & -2.337 & 0.392 & 35.493 & $<0.001$ & $0.10(0.05-0.21)$ \\
Constant & -2.798 & 0.202 & 191.550 & $<0.001$ & 0.061 \\
\hline
\end{tabular}

Nagelkerke $R^{2}=7.7 \%$. Area under the curve $=0.672$

ACR American College of Radiology; CI confidence interval
Table 4 Logistic regression model of variables associated with reoperation

Table 5 Logistic regression model of variables associated with overall mastectomy

\begin{tabular}{|c|c|c|c|c|c|}
\hline Variable & $\mathrm{T}$ & Standard error & Wald & $p$ & $\begin{array}{l}\text { Odds ratio } \\
(95 \% \mathrm{CI})\end{array}$ \\
\hline MRI & 1.472 & 0.170 & 75.022 & $<0.001$ & $4.36(3.12-6.08)$ \\
\hline High familial risk ${ }^{\mathrm{a}}$ & 1.372 & 0.498 & 7.599 & 0.006 & $3.95(1.49-10.47)$ \\
\hline Premenopausal & 0.478 & 0.155 & 9.571 & 0.002 & $1.61(1.19-2.18)$ \\
\hline ACR breast density category $b$ & 0.539 & 0.253 & 4.529 & 0.033 & $1.72(1.04-2.82)$ \\
\hline ACR breast density category $c$ & 0.423 & 0.266 & 2.523 & 0.112 & $1.53(0.91-2.57)$ \\
\hline ACR breast density category $d$ & 0.927 & 0.313 & 8.761 & 0.003 & $2.53(1.37-4.67)$ \\
\hline Lobular histology & 0.467 & 0.178 & 6.859 & 0.009 & $1.60(1.13-2.26)$ \\
\hline Tumor size on final pathology $\geq 20 \mathrm{~mm}$ & 0.738 & 0.140 & 27.926 & $<0.001$ & $2.09(1.59-2.75)$ \\
\hline Planned mastectomy on conventional imaging & 6.386 & 0.716 & 79.616 & $<0.001$ & $594.65(146.00-2414.15)$ \\
\hline Constant & -4.128 & 0.261 & 249.319 & $<0.001$ & 0.016 \\
\hline
\end{tabular}

Nagelkerke $R^{2}=59.4 \%$. Area under the curve $=0.884$

ACR American College of Radiology; CI confidence interval; MRI magnetic resonance imaging

a Three or more first-degree relatives with breast or ovarian cancer

the assessment of breast recurrence and distant metastases rates.

The strengths of this observational study are related to its real-world multicenter large-scale size [30], which allowed us to take into consideration real-world data on 5896 patients. The real-world data approach of the MIPA study reflects current clinical practice, providing insights on a variety of issues, frequently overlooked in the discussion about preoperative MRI, such as the selection of patients referred for preoperative MRI and the difficulties in obtaining a lesion-by-lesion radiological-pathological correlation in everyday practice.

The first limitation of this study, i.e., its non-randomized design, is indeed counteracted by the approach of the MIPA study with a large and diverse study population. Other limitations of the study are represented by the 
exclusion of 1349 patients from analysis and the selection of centers with considerable clinical experience and high breast MRI volumes, potentially limiting the generalizability of our results.

Notwithstanding the aforementioned limitations, the results of our study - which provides quantitative information about the probability of conversion from breastconserving surgery to mastectomy and the reduction of the reoperation rate-may be usefully taken into account in tumor board meetings and may contribute to enhance the awareness and involvement of breast cancer patients when MRI is being considered before surgery.

In conclusion, the MIPA study evaluated the clinical practice of performing or not performing breast MRI in a large cohort of breast cancer patients. Across 27 centers worldwide, $53 \%$ of patients underwent MRI, with a 4.4 OR of receiving mastectomy when compared to patients who underwent conventional imaging only, counterbalanced by a 3\% lower reoperation rate. Mastectomy was already planned on the basis of conventional imaging in $14 \%$ of the noMRI group and $22 \%$ of the MRI group, where MRI was frequently used as a confirmation tool toward mastectomy, leading to an $11 \%$ increase in mastectomy rate, $9 \%$ when excluding patient's preference.

Acknowledgements The MIPA study was promoted by the European Network for the Assessment of Imaging in Medicine (EuroAIM), a joint initiative of the European Institute for Biomedical Imaging Research (EIBIR), and endorsed by the European Society of Breast Imaging. The authors thank Bayer AG that provided an unconditional research grant, in particular Dr. Stephanie Schermuck-Joschko (who passed away due to a car accident after the study started) and Dr. Jan Endrikat. The authors also thank Monika Hierath, Eva Haas, Katharina Krischak, and Peter Gordebeke from the EIBIR staff for the management of all the administrative work of this study. The following persons collaborated at individual centres: Lucia Camera, MD, Department of Radiology, Azienda Ospedaliera Universitaria Integrata, Verona, Italy; Sara Mirandola, MD, Department of Surgery, Azienda Ospedaliera Universitaria Integrata, Verona, Italy; Marta Maria Panzeri, MD, Department of Breast Radiology, IRCCS Ospedale San Raffaele, Milan, Italy; Carolina Rossi Saccarelli, MD, Maria A. Rodi Carvalho Barros Bernardes, MD, Vera L. Nunes Aguillar, MD, PhD (all three from the Department of Radiology), and Alfredo Carlos S. D. Barros, MD, PhD (Department of Breast Surgery), Hospital Sírio Libanês, São Paulo, Brazil; Katja Siegmann-Luz, MD, and Benjamin Wiesinger, MD, Department of Diagnostic and Interventional Radiology, University Hospital of Tübingen, Germany; James M. Anderson, Max Hobbs, and Wanda Gunawan, Royal Perth Hospital, Perth, Australia.

Funding Open access funding provided by Università degli Studi di Milano within the CRUI-CARE Agreement. This study received an unconditional research grant from Bayer AG. This company did not have any influence on the study protocol planning, did not have any access to the study database, and was not involved in any way in the data analysis, manuscript writing, or submission phases. Among authors, Fiona J. Gilbert and Sarah Hilborne were supported by the NIHR Cambridge Biomedical Research Centre (BRC-1215-20014). The views expressed are those of the authors and not necessarily those of the NIHR or the UK Department of Health and Social Care.

\section{Declarations}

Guarantor The scientific guarantor of this publication is Prof. Francesco Sardanelli, MD.

Conflict of interest Outside the present work, the authors declare the following relations with companies and institutions:

Francesco Sardanelli received research grants from - and is a member of the speakers' bureau of - General Electric Healthcare, Bayer, and Bracco; he is also a member of the Bracco Advisory Group.

Nehmat Houssami receives research funding via a National Breast Cancer Foundation (NBCF Australia) Breast Cancer Research Leadership Fellowship.

Fiona J. Gilbert received research grants from General Electric Healthcare, GSK and Hologic, and had research collaborations with Volpara and Bayer.

Marc B.I. Lobbes received a research grant and is a member of the speakers' bureau of General Electric Healthcare.

Katja Pinker declares funding by the NIH/NCI Cancer Centre Support Grant P30 CA008748, Digital Hybrid Breast PET/MRI for Enhanced Diagnosis of Breast Cancer (HYPMED), H2020 - Research and Innovation Framework Programme PHC-11-2015 \# 667211-2, A Body Scan for Cancer Detection using Quantum Technology (CANCERSCAN), H2020-FETOPEN-2018-2019-2020-01 \# 828978, Multiparametric 18F-Fluoroestradiol PET/MRI coupled with Radiomics Analysis and Machine Learning for Prediction and Assessment of Response to Neoadjuvant Endocrine Therapy in Patients with Hormone Receptor+l HER2- Invasive Breast Cancer 02.09.2019/31.08.2020 \# Nr: 18207, Jubiläumsfonds of the Austrian National Bank.

Simone Schiaffino received travel support from Bracco Imaging and is a member of the speakers' bureau for General Electric Healthcare. All other authors declare that they have no conflict of interest related to the present work and that they have nothing to disclose.

Statistics and biometry The third author (Nehmat Houssami, The University of Sydney) and the last author (Giovanni Di Leo, IRCCS Policlinico San Donato) have significant statistical expertise.

Informed consent Written informed consent was obtained from all patients in this study, unless waived by local Ethics Committees.

Ethical approval This study was approved by the Ethics Committee of the coordinating centre on January 29, 2013 (protocol number 2784) and thereafter by local Ethics Committees of participating centres.

\section{Methodology}

Prospective

Observational

Multicentre study

Open Access This article is licensed under a Creative Commons Attribution 4.0 International License, which permits use, sharing, adaptation, distribution and reproduction in any medium or format, as long as you give appropriate credit to the original author(s) and the source, provide a link to the Creative Commons licence, and indicate if changes were made. The images or other third party material in this article are included in the article's Creative Commons licence, unless indicated otherwise in a credit line to the material. If material is not included in the article's Creative Commons licence and your intended use is not permitted by statutory regulation or exceeds the permitted use, you will need to obtain permission directly from the copyright holder. To view a copy of this licence, visit http://creativecommons.org/licenses/by/4.0/. 


\section{References}

1. Lehman CD, Gatsonis C, Kuhl CK et al (2007) MRI evaluation of the contralateral breast in women with recently diagnosed breast cancer. N Engl J Med 356:1295-1303. https://doi.org/10.1056/ NEJMoa065447

2. Ray KM, Hayward JH, Joe BN (2018) Role of MR imaging for the locoregional staging of breast cancer. Magn Reson Imaging Clin N Am 26:191-205. https://doi.org/10.1016/j.mric.2017.12.008

3. Houssami N, Turner R, Macaskill P et al (2014) An individual person data meta-analysis of preoperative magnetic resonance imaging and breast cancer recurrence. J Clin Oncol 32:392-401. https://doi.org/10.1200/JCO.2013.52.7515

4. Houssami N, Turner RM, Morrow M (2017) Meta-analysis of pre-operative magnetic resonance imaging (MRI) and surgical treatment for breast cancer. Breast Cancer Res Treat 165:273-283. https://doi.org/10.1007/s10549-017-4324-3

5. Monticciolo DL (2017) Practical considerations for the use of breast MRI for breast cancer evaluation in the preoperative setting. Acad Radiol 24:1447-1450. https://doi.org/10.1016/j.acra.2017. 05.012

6. Sardanelli F, Newstead GM, Putz B et al (2016) Gadobutrolenhanced magnetic resonance imaging of the breast in the preoperative setting. Invest Radiol 51:454-461. https://doi.org/10.1097/ RLI.0000000000000254

7. Plana MN, Carreira C, Muriel A et al (2012) Magnetic resonance imaging in the preoperative assessment of patients with primary breast cancer: systematic review of diagnostic accuracy and meta-analysis. Eur Radiol 22:26-38. https://doi.org/10.1007/ s00330-011-2238-8

8. Jatoi I, Benson JR (2013) The case against routine preoperative breast MRI. Futur Oncol 9:347-353. https://doi.org/10.2217/fon.12.186

9. Arnaout A, Catley C, Booth CM et al (2015) Use of preoperative magnetic resonance imaging for breast cancer. JAMA Oncol 1:1238-1250. https://doi.org/10.1001/jamaoncol.2015.3018

10. Zeng Z, Amin A, Roy A et al (2020) Preoperative magnetic resonance imaging use and oncologic outcomes in premenopausal breast cancer patients. NPJ Breast Cancer 6:49. https://doi.org/10. 1038/s41523-020-00192-7

11. Sardanelli F, Boetes C, Borisch B et al (2010) Magnetic resonance imaging of the breast: Recommendations from the EUSOMA working group. Eur J Cancer 46:1296-1316. https://doi.org/10. 1016/j.ejca.2010.02.015

12. Mann RM, Balleyguier C, Baltzer PA et al (2015) Breast MRI: EUSOBI recommendations for women's information. Eur Radiol 25:3669-3678. https://doi.org/10.1007/s00330-015-3807-z

13. The American Society of Breast Surgeons (2016) Don't routinely order breast MRI in new breast cancer patients. The American Society of Breast Surgeons, Columbia, MD, USA. Available via https://www.choosingwisely.org/societies/american-society-ofbreast-surgeons/. Accessed 01 Jun 2021

14. Sardanelli F, Trimboli RM, Houssami N et al (2020) Solving the preoperative breast MRI conundrum: design and protocol of the MIPA study. Eur Radiol 30(10):5427-5436. https://doi.org/10. 1007/s00330-020-06824-7

15. Newell MS, Giess CS, Argus AD et al (2018) ACR practice parameter for the performance of contrast-enhanced magnetic resonance imaging (MRI) of the breast. American College of Radiology, Reston, VA, USA. Available via https://www.acr.org/-/media/ACR/Files/PracticeParameters/MR-Contrast-Breast.pdf. Accessed 01 Jun 2021

16. Parker A, Schroen AT, Brenin DR (2013) MRI utilization in newly diagnosed breast cancer: a survey of practicing surgeons. Ann Surg Oncol 20:2600-2606. https://doi.org/10.1245/s10434-013-2934-5

17. Lee J, Tanaka E, Eby PR et al (2017) Preoperative breast MRI: surgeons' patient selection patterns and potential bias in outcomes analyses. AJR Am J Roentgenol 208:923-932. https://doi.org/10. 2214/AJR.16.17038

18. McLaughlin SA (2013) Surgical management of the breast. Surg Clin North Am 93:411-428. https://doi.org/10.1016/j.suc.2012.12.006

19. Margenthaler JA, Ollila DW (2016) Breast conservation therapy versus mastectomy: shared decision-making strategies and overcoming decisional conflicts in your patients. Ann Surg Oncol 23:3133-3137. https://doi.org/10.1245/s10434-016-5369-y

20. Tot T, Gere M (2008) Radiological-pathological correlation in diagnosing breast carcinoma: the role of pathology in the multimodality era. Pathol Oncol Res 14:173-178. https://doi.org/10.1007/ s12253-008-9061-9

21. Gonzalez V, Sandelin K, Karlsson A et al (2014) Preoperative MRI of the breast (POMB) influences primary treatment in breast cancer: a prospective, randomized, multicenter study. World J Surg 38:1685-1693. https://doi.org/10.1007/s00268-014-2605-0

22. Balleyguier C, Dunant A, Ceugnart L et al (2019) Preoperative breast magnetic resonance imaging in women with local ductal carcinoma in situ to optimize surgical outcomes: results from the randomized phase III trial IRCIS. J Clin Oncol 37:885-892. https:// doi.org/10.1200/JCO.18.00595

23. Walsh SM, Brennan SB, Zabor EC et al (2019) Does breast density increase the risk of re-excision for women with breast cancer having breast-conservation therapy? Ann Surg Oncol 26:4246-4253. https://doi.org/10.1245/s10434-019-07647-7

24. McCahill LE, Single RM, Aiello Bowles EJ et al (2012) Variability in reexcision following breast conservation surgery. JAMA 307:467-475. https://doi.org/10.1001/jama.2012.43

25. Landercasper J, Borgert AJ, Fayanju OM et al (2019) Factors associated with reoperation in breast-conserving surgery for cancer: a prospective study of american society of breast surgeon members. Ann Surg Oncol 26:3321-3336. https://doi.org/10.1245/ s10434-019-07547-w

26. Turnbull L, Brown S, Harvey I et al (2010) Comparative effectiveness of MRI in breast cancer (COMICE) trial: a randomised controlled trial. Lancet 375:563-571. https://doi.org/10.1016/ S0140-6736(09)62070-5

27. Peters NHGM, van Esser S, van den Bosch MAAJ et al (2011) Preoperative MRI and surgical management in patients with nonpalpable breast cancer: The MONET - Randomised controlled trial. Eur J Cancer 47:879-886. https://doi.org/10.1016/j.ejca.2010. 11.035

28. Heil J, Breitkreuz K, Golatta M et al (2012) Do reexcisions impair aesthetic outcome in breast conservation surgery? exploratory analysis of a prospective cohort study. Ann Surg Oncol 19:541-547. https://doi.org/10.1245/s10434-011-1947-1

29. Olsen MA, Nickel KB, Margenthaler JA et al (2015) Increased risk of surgical site infection among breast-conserving surgery reexcisions. Ann Surg Oncol 22:2003-2009. https://doi.org/10.1245/ s10434-014-4200-x

30. Nabhan C, Klink A, Prasad V (2019) Real-world evidence-what does it really mean? JAMA Oncol 5:781-783. https://doi.org/10. 1001/jamaoncol.2019.0450

Publisher's note Springer Nature remains neutral with regard to jurisdictional claims in published maps and institutional affiliations.

Rubina M. Trimboli is now at the Breast Imaging and Screening Unit, Department of Radiology, Humanitas Clinical and Research Center IRCCS, Rozzano, Italy; Julia Camps Herrero is now at Área de Salud de la Mama, Ribera Salud Grupo, Valencia, Spain; Gábor Forrai is now at the Department of Radiology, Duna Medical Center, Budapest, Hungary; and Marc B. I. Lobbes is now at the Department of Radiology, Zuyderland Medical Center, Sittard-Geleen, The Netherlands. 


\section{Authors and Affiliations}

Francesco Sardanelli ${ }^{1,2}$ (D) Rubina M. Trimboli ${ }^{1}$. Nehmat Houssami ${ }^{3} \cdot$ Fiona J. Gilbert $^{4} \cdot$ Thomas H. Helbich $^{5}$. Marina Álvarez Benito ${ }^{6}$. Corinne Balleyguier ${ }^{7}$. Massimo Bazzocchi ${ }^{8} \cdot$ Peter Bult $^{9} \cdot$ Massimo Calabrese $^{10}$. Julia Camps Herrero ${ }^{11}$. Francesco Cartia ${ }^{12}$ - Enrico Cassano ${ }^{13}$. Paola Clauser ${ }^{5}$. Andrea Cozzi ${ }^{1}$. Danúbia A. de Andrade ${ }^{14}$. Marcos F. de Lima Docema ${ }^{15}$. Catherine Depretto ${ }^{12}$. Valeria Dominelli ${ }^{13}$. Gábor Forrai ${ }^{16}$. Rossano Girometti ${ }^{8}$. Steven E. Harms ${ }^{17}$. Sarah Hilborne ${ }^{4}$. Raffaele lenzi ${ }^{18}$. Marc B. I. Lobbes ${ }^{19}$. Claudio Losio ${ }^{20}$. Ritse M. Mann ${ }^{21,22}$. Stefania Montemezzi ${ }^{23}$. Inge-Marie Obdeijn ${ }^{24}$. Umit A. Ozcan ${ }^{25}$. Federica Pediconi ${ }^{26}$. Katja Pinker,27 . Heike Preibsch ${ }^{28}$. José L. Raya Povedano ${ }^{6}$. Daniela Sacchetto ${ }^{29,30,31}$. Gianfranco P. Scaperrotta ${ }^{12}$. Simone Schiaffino ${ }^{2} \cdot$ Margrethe Schlooz $^{32}$ - Botond K. Szabó ' $^{33}$. Donna B. Taylor ${ }^{34,35}$. Özden S. Ulus ${ }^{25}$. Mireille Van Goethem ${ }^{36}$. Jeroen Veltman ${ }^{37}$. Stefanie Weigel ${ }^{38} \cdot$ Evelyn Wenkel $^{39} \cdot$ Chiara Zuiani $^{8} \cdot$ Giovanni Di Leo $^{2}$

1 Department of Biomedical Sciences for Health, Università degli Studi di Milano, Milan, Italy

2 Unit of Radiology, IRCCS Policlinico San Donato, Via Rodolfo Morandi 30, 20097 San Donato Milanese, Italy

3 Sydney School of Public Health, Faculty of Medicine and Health, The University of Sydney, Sydney, Australia

4 Department of Radiology, School of Clinical Medicine, Cambridge Biomedical Campus, University of Cambridge, Cambridge, UK

5 Department of Biomedical Imaging and Image-guided Therapy, Division of General and Pediatric Radiology, Research Group: Molecular and Gender Imaging, Medical University of Vienna, Vienna, Austria

6 Department of Radiology, Hospital Universitario Reina Sofía, Córdoba, Spain

7 Department of Radiology, Institut Gustave Roussy, Villejuif, France

8 Institute of Radiology, Department of Medicine, Università degli Studi di Udine, Udine, Italy

9 Department of Pathology, Radboud University Medical Center, Nijmegen, The Netherlands

10 Unit of Breast Radiology, IRCCS Ospedale Policlinico San Martino, Genoa, Italy

11 Department of Radiology, Hospital Universitario de La Ribera, Alzira, Spain

12 Unit of Breast Imaging, Fondazione IRCCS Istituto Nazionale dei Tumori, Milan, Italy

13 Breast Imaging Division, IEO, European Institute of Oncology IRCCS, Milan, Italy

14 Department of Breast Surgery, Hospital Sírio Libanês, São Paulo, Brazil

15 Department of Radiology, Hospital Sírio Libanês, São Paulo, Brazil

16 Department of Radiology, MHEK Teaching Hospital, Semmelweis University, Budapest, Hungary

17 Breast Center of Northwest Arkansas, Fayetteville, AR, USA
18 Department of Radiology, Di.Bi.MED, Università degli Studi di Palermo, Policlinico Universitario Paolo Giaccone, Palermo, Italy

19 Department of Radiology and Nuclear Medicine, Maastricht University Medical Center, Maastricht, The Netherlands

20 Department of Breast Radiology, IRCCS Ospedale San Raffaele, Milan, Italy

21 Department of Radiology and Nuclear Medicine, Radboud University Medical Center, Nijmegen, The Netherlands

22 Department of Radiology, The Netherlands Cancer Institute, Amsterdam, The Netherlands

23 Department of Radiology, Azienda Ospedaliera Universitaria Integrata Verona, Verona, Italy

24 Department of Radiology and Nuclear Medicine, Erasmus University Medical Center, Rotterdam, The Netherlands

25 Unit of Radiology, Acıbadem Mehmet Ali Aydınlar University School of Medicine, İstanbul, Turkey

26 Department of Radiological, Oncological and Pathological Sciences, Università degli Studi di Roma "La Sapienza", Rome, Italy

27 Department of Radiology, Breast Imaging Service, Memorial Sloan Kettering Cancer Center, New York, NY, USA

28 Department of Diagnostic and Interventional Radiology, University Hospital of Tübingen, Tübingen, Germany

29 Kiwifarm S.R.L, La Morra, Italy

30 Disaster Medicine Service 118, ASL CN1, Saluzzo, Italy

31 CRIMEDIM, Research Center in Emergency and Disaster Medicine, Università degli Studi del Piemonte Orientale "Amedeo Avogadro", Novara, Italy

32 Department of Surgery, Radboud University Medical Center, Nijmegen, The Netherlands

33 Department of Radiology, Barking Havering and Redbridge University Hospitals NHS Trust, London, UK

34 Medical School, Faculty of Health and Medical Sciences, The University of Western Australia, Perth, Australia 
35 Department of Radiology, Royal Perth Hospital, Perth, Australia

36 Gynecological Oncology Unit, Department of Obstetrics and Gynecology, Department of Radiology, Multidisciplinary Breast Clinic, Antwerp University Hospital, University of Antwerp, Antwerpen, Belgium

37 Maatschap Radiologie Oost-Nederland, Oldenzaal, The Netherlands
38 Institute of Clinical Radiology and Reference Center for Mammography, University of Münster, Münster, Germany

39 Department of Radiology, University Hospital of Erlangen, Erlangen, Germany 